\title{
D2D Technology Implementation in 5G Network and the Security Aspect: A Review
}

\author{
Samir Čaušević, Adisa Medić, Nedžad Branković \\ Faculty of Traffic and Communications, University of Sarajevo, Sarajevo, Bosnia and Herzegovina
}

\begin{abstract}
D2D is a promising paradigm of the 5G network offering a beneficial infrastructure and allowing different applications such as social applications, advertising services, or those supporting mutual connectivity of heterogenous objects, as well as those offering support in cases of natural disasters.

Compared to conventional network communication, communication achieved by D2D technology shows a lower degree of security. It is necessary to modify existing security mechanisms and new solutions to be adapted to the application of D2D in the fifth generation network, which will raise the level of security in terms of authenticity verification, ensuring service availability and maintaining integrity.
\end{abstract}

Keywords - 5G network, D2D communication, security, threats and attacks, security-related solutions

\section{Introduction}

The growing demand for a greater speed of data transfer, minimum delay, coverage and great reliability requires one look for solutions aimed at an integration of $2 \mathrm{G}, 3 \mathrm{G}, 4 \mathrm{G}$ and other access technologies which, in fact, the $5 \mathrm{G}$ network enables.

DOI: 10.18421/TEM102-64

https://doi.org/10.18421/TEM102-64

Corresponding author: Adisa Medić,

Faculty of Traffic and Communications, University of Sarajevo, Sarajevo, Bosnia and Herzegovina.

Email: $\underline{\text { s adisa@hotmail.com }}$

Received: 02 March 2021.

Revised: 12 May 2021.

Accepted: 19 May 2021.

Published: 27 May 2021.

(cc)BY-Nc-ND (c) 2021 Samir Čaušević, Adisa Medić \& Nedžad Branković; published by UIKTEN. This work is licensed under the Creative Commons AttributionNonCommercial-NoDerivs 4.0 License.

The article is published with Open Access at www.temjournal.com
D2D communication has been proposed as an instrument for utilizing the physical proximity of communication devices [1], and together with other technologies of the fifth generation $(5 \mathrm{G})$ network represents a testing ground for new ideas in the progress of people's lives. Communication between devices in D2D will be possible by direct interconnection and data sharing without an indirect connection to the base station, as well as via user equipment acting as relays [2].

IoT (Internet of Things) is a concept of $5 \mathrm{G}$ network should provide connection and interaction of different objects and thus turn our cities, houses, vehicles into smart and interconnected, to which D2D technology contributes greatly[3].

D2D functionality supports high baud rates and reduces latency between devices, making it particularly important in meeting the standards set for $5 \mathrm{G}$ network deployment. In the cloud-based mobile computer technology, it allows for a more efficient sharing or resources (spectrum, calculation power, applications, social content, etc.) for users which are spatially proximal to each other. The second part of the present paper discusses the very term, the features and basic characteristics of D2D communication, whereas the third part encompasses and describes the fields of D2D communication implementation in the $5 \mathrm{G}$ network.

When it comes to the security aspect, the fourth chapter of the present paper offers a detailed insight into and an analysis of threats and attacks, such as fake authorship, DoD, MITMA, eavesdropping attacks, etc. as well as security-related to solutions as counter-measures.

\section{D2D Communication - the Term and Its Main Features}

The communication between devices and D2D devices (in further text: D2D) presents one of the most promising perspectives of wireless communication technologies within the new 5G mobile communication network, which allows a direct communication between physically proximal devices and the data exchange service [4], [5]. The scope of the application of D2D communication has expanded and it is expected that billions of devices will be communicating in the years to come. 
The main feature that makes the D2D communications different in relation to other communications based on traditional infrastructure is the limited involvement of the Base Station in communication or a complete lack of the central part such as is the Access Point or the Base Station, where the data between a pair of devices should not be passing through the core of the network as long as their distance is proximal.
In [6], a two-layer 5G cellular network is planned and it implies the macrocellular level in which the communication between the devices is maintained by means of the Base Station and the level of the device related to the direct connection with another device or enabling transmission by means of other devices. Therefore, four types of communication at the device level are identified as the picture below shows:

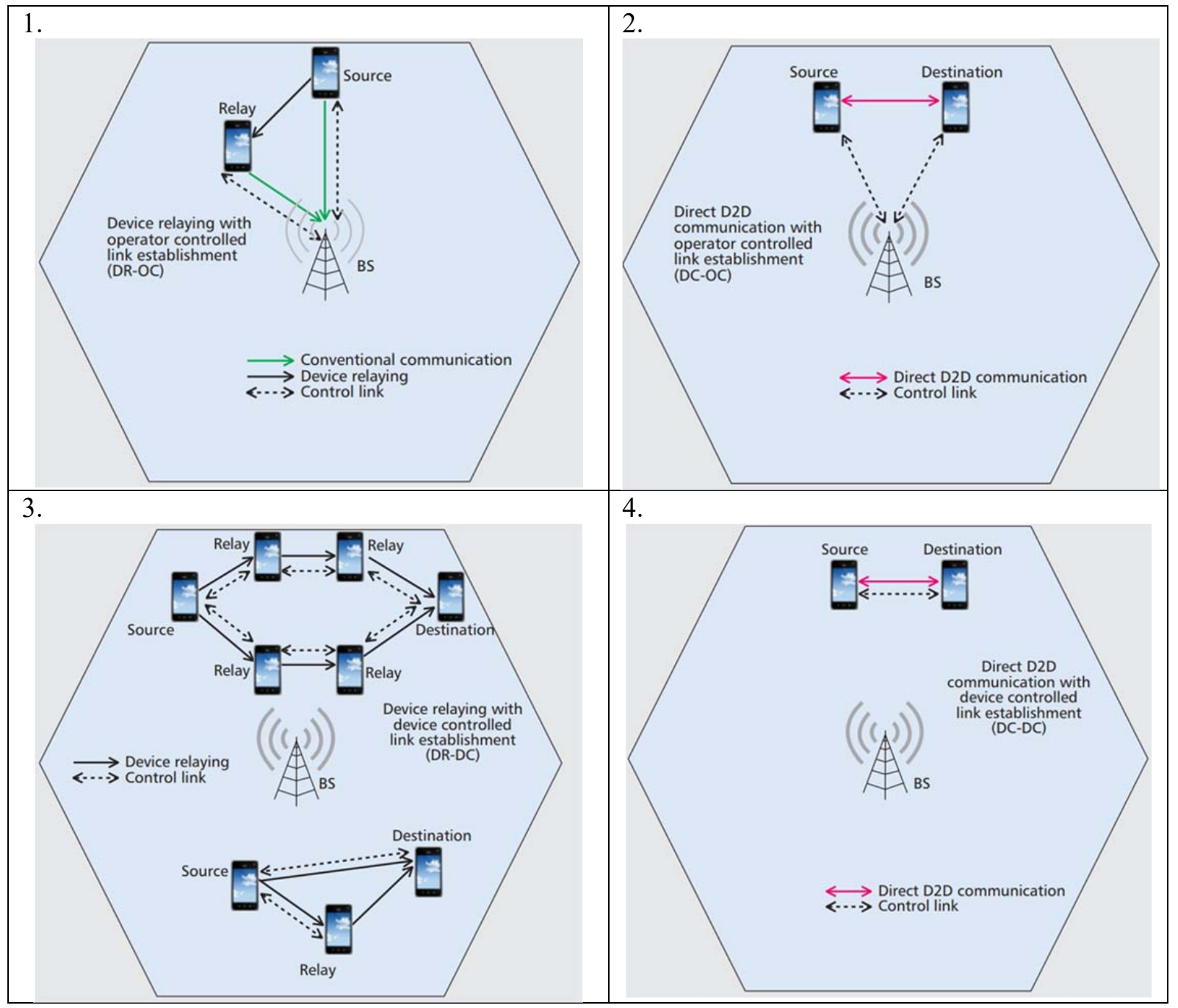

Figure 1. An illustration of D2D communication

The first part of the Figure 1 is a graphical representation of the established connection of the device with the Base Station through the use of another device as a relay, menages by the operator. As in the first part of the image, the second part is about communication that is completely controlled by the Base Station. The difference is that the connection between the devices is established directly.

The third type of communication, graphically represented by the third part of the image, implies controlling the connection by the devices themselves, without inclusion the Base Station, which is communicating via relays. The fourth part of the image shows how devices communicate directly without assistance of the base station.

Currently, on the market, technologies such as WIFI and Bluetooth offer some D2D communications functions. However, they work in a non-licensed scope and the interference in uncontrolled. Besides, they cannot offer security or the quality of service (QoS) which applies to cellular networks up until present [6].

D2D classification can be illustrated as in Figure 2 according to [7], [32],[8]: 


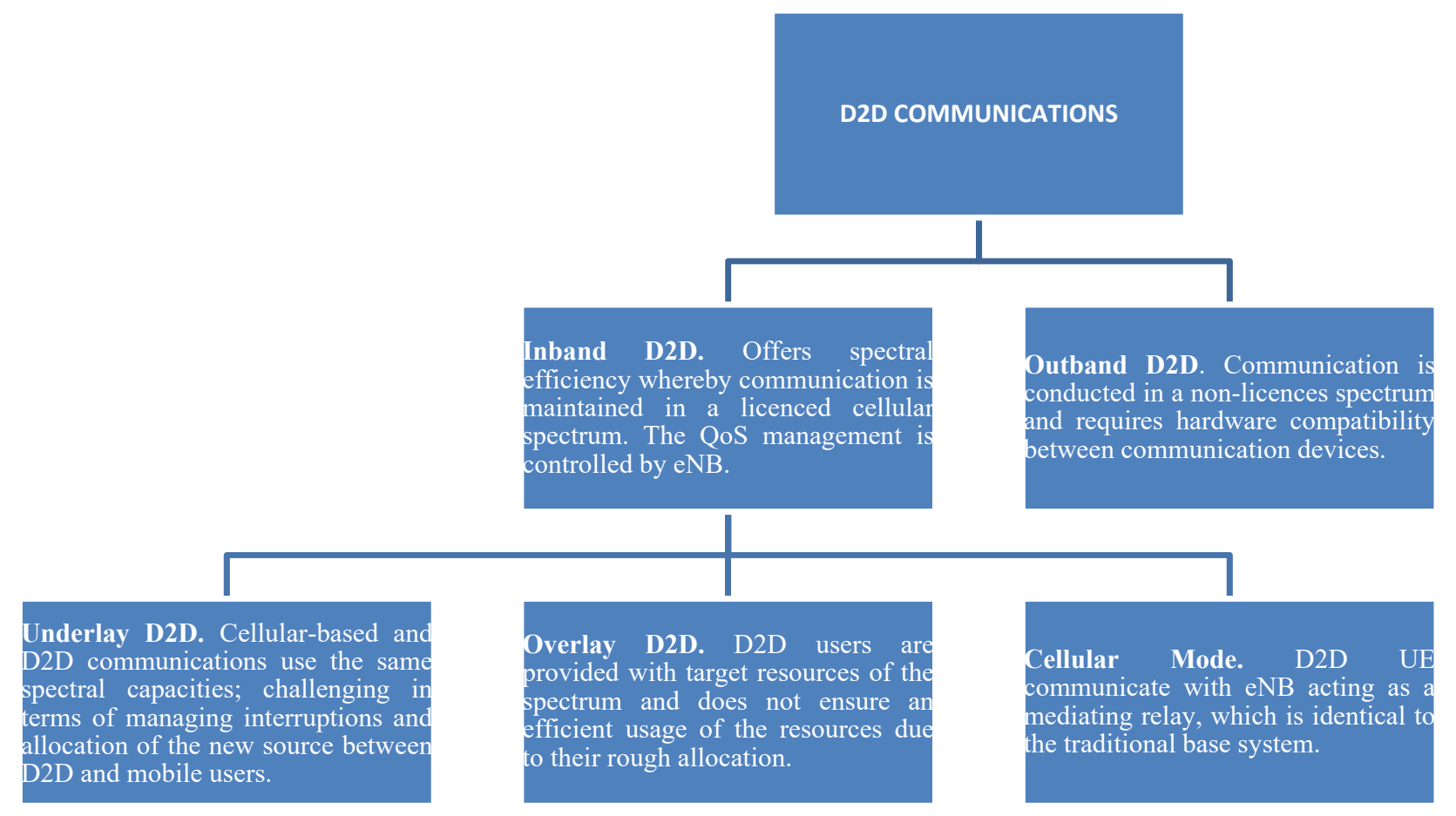

Figure 2. D2D communication classification

By implementing D2D technology in 5G, aside from the goals pertaining to end-to-end security and the reduction of costs, the following advantages [9], [33] are obtained in terms of:

1. Better cell coverage through the establishment of communication between the device and the base station via an appropriate relay;

2. Great speed of data transmission supported by devices located further from BS/ AP;

3. Reliable communication and in the case of the network breakdown, which may be a disaster scenario;

4. Radiofrequency interruptions are reduced in the D2D communication scenario, and it assists in the transmission of multifold data using the same radio frequency;

5. It is higher energy efficiency of the base stations;

6. A reduction of traffic that reduced the total number of cellular connections;

7. Heterogenous linkage, taking into consideration that direct communication between devices does not rely only onto the base interface but may be established by means of alternative radio technologies;

8. Ultra-low latency in communications due to a shorter path of signal transmission.

\section{Application of D2D Technology in 5G Network}

5G network comprises an implementation of a heterogenous network (HetNet) by means of using different technologies of radio access, multifold backhaul technique and station hierarchy - macro, pico, and femto cells, great arrays of antennae on the BS serving a great number of users (massive MIMO), cognitive radio networks (CRN) on which the secondary users "opportunistically" use the primary user spectrum, mm Wave spectrum and D2D communication [10].

For enabling D2D connections of short range, millimetre wave spectrum of $5 \mathrm{G}$ network is used, whereas secondary users in CRN can also use D2D communication in order to avoid interruptions for primary users. In terms of enhancing spectrum efficiency and data transmission speed, D2D communication adds to the HetNets and massive MIMO of the enabled BS. In addition, MIMO antennae built into user devices enhance resistance to noise and the system capacity by using the varieties and by multiplexing gains [11].

A new trend in D2D communication is introducing sensors and sensor-based systems, while 5G may expand IoT possibilities founded on sensors by means of introducing robots, actuators and drones for a distributional co-ordination and reliable performances with little delay [12].

D2D networks can be used for establishing M2M communication in IoT as they enable responses in real time and provide an ultra-low latency [13]. Generally, the problem in cellular communication is that it requires great energy consumption. An implementation of IoT in the smart city by introducing D2D communication is possible due to the efficient usage of radio source and a small energy consumption characterized by D2D [14]. This feature 
of the $5 \mathrm{G}$ network will also find its application in vehicle-to-vehicle (V2V) communication in terms of data sharing between neighbouring vehicles and efficient offload traffic. D2D can also be used for the communication from the vehicle to an infrastructure and from the vehicle to the pedestrian. The stated area of application of D2D technology in the 5G network together with other areas defined in Chapters 3.1., 3.2. and 3.3. can be represented by Figure 3.

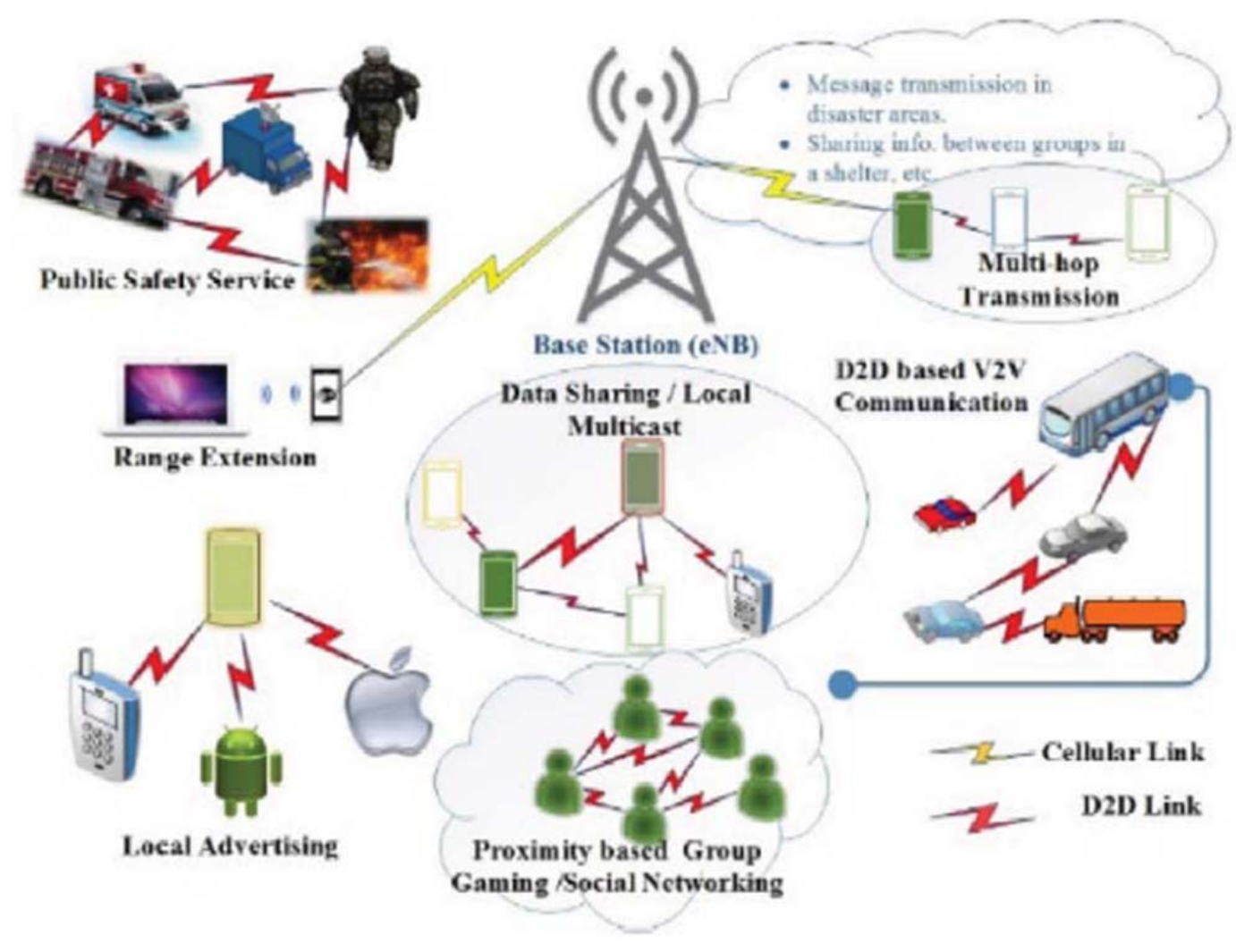

Figure 3. Applications of D2D communication [15]

The implementation of D2D technology in 5G network can also be discussed at the local service level, communication in case of emergency and in terms of IoT improvement, which will be discussed in further text.

\subsection{Local Service}

Within the local service, user data are transmitted directly between the terminals and do not include the network side, e.g. applications for social media that are based on the service of proximity [16], [17]. Social applications present the base D2D application, where the users, on the basis of functions of finding access and communication may find each other, use social applications and exchange data.

A new source of income for operators may be ensured through a local data transfer (e.g., advertising services) where a mall can send advertisements, discounts and promotions to the people who enter the mall or walk around it, whereas a nearby cinema, for instance, may forward information about its movie projections.
Services such as HD video recording set high goals for the base network and the spectrum resources, whereby D2D can assist in easing cellular traffic and reducing the transmission pressure of cellular networks. At hot spots, operators or service providers can set up media providers that store popular media services. These media providers offer media services in a D2D fashion. Users can use D2D for obtaining media content from the nearby user terminals which have received media services.

\subsection{Emergency Communication}

D2D communication may be of crucial importance in natural disasters. In cases of earthquakes or hurricanes, the communication networks infrastructure can be damaged and the network may even collapse [8]. By means of D2D functionality, an emergency communication network may be set up as a replacement for the damaged communications networks and internet infrastructures. By means of D2D with one or more jumbs, the users in dead ends may be connected by user terminals which are found in the areas of coverage, and then connected to the wireless network. 


\subsection{IoT Improvement}

IoT represents an integration of heterogenous objects of various possibilities of connectivity and calculation, with various types of interaction, including communication between machines, and machines and people [18].

A typical application of IoT D2D-based improvement is vehicle-to-vehicle communication (V2V) on the Internet. In case it is moving at a great speed, the vehicle may be warned about the presence of other nearby vehicles by means of D2D before it moves the lane or slows down [8]. Based on the warnings received, the nearby vehicles warn the drivers or even automatically control driving in extraordinary situations in order for the drivers to be able to react faster and in order to reduce the number of traffic accidents. Furthermore, by means of D2Dobtained information, vehicles can reliably detect and identify certain nearby vehicles, such as those that can present a danger at cross-sections or vehicles that require special attention (e.g., school buses and vehicle transporting dangerous goods).

D2D may also assist in solving issues in new scenarios of wireless communication. In the internal positioning, terminals cannot get satellite signals when they are in a closed space. In this case, traditional satellite positioning does not function. However, in the internal D2D-based positioning, the earlier set-up terminals with given information about the location or the usual external terminals with a given location can determine the place of the terminals that need to be positioned and support internal positioning in $5 \mathrm{G}$ networks at a low cost.

\section{The Security Aspect of D2D Communication Implemented in 5G Network}

Despite the advanced features and the implementation of the 5th generation mobile networks, D2D technology has been facing numerous technical challenges and issues related to device detection, control of power charging, selection of methods, managing resources and interruptions, mobility, interference and the questions related to security and privacy [19].
In this part of the paper, a detailed account of the security-related issues is given when it comes to D2D communication of the $5 \mathrm{G}$ network by presenting security threats and potential security solutions for them. In order for the D2D network to ensure the security aspect, it needs to meet the functions and requirements of integrity, availability, reliability, anonymity, non-connectedness, safe directioning and transmission, confidentiality and authentication [5].

\subsection{D2D and Security Threats}

The demands of users for great data transfer speeds, low delay and a longer network coverage have brought to the introduction of $5 \mathrm{G}$ technology of multiple entry points, multiple exit points and communication among devices where, in terms of providing longer coverage, the network is being introduced by new wireless components such as relays, smart and small access points and hotspots.

In the case when the user device is not found within the range of base station, the communication with the base station is realized through the transmission of messages via the interdevices-relay. Relay communication has advantages that are reflected in expanding the coverage of the cellular network and improving the quality of service, while on the other hand it is sensitive in terms of data protection from unauthorized access, and intentional or accidental unauthorized modification [20].

There are three phases in the realization of information communication between D2D: device detection, establishing the connection and data transmission by means of a direct link between devices. In the detection phase, the devices find each other and establish a direct connection, followed by the data transfer phase. Since in the phase during which the connection is being established, there is no procedure of authenticity for the verification of the identity of the device, the attacker may carry out attacks about which more is to be said in the present paper.

As data in $\mathrm{D} 2 \mathrm{D}$ communication of the $5 \mathrm{G}$ network is transmitted over the interdevices, new possibilities of potential security vulnerabilities such as data hacking grow, which means a violation of privacy and confidentiality [21].

The attacks may be classified on the basis of access control, accessibility, authenticity, confidentiality, and integrity [22]. Furthermore, a detailed review of D2D is given in the Table 1 below as follows: 
Table 1. Security threats of D2D communication [23], [24], [5]

\begin{tabular}{|c|c|}
\hline Security D2D threat & Security threat feature \\
\hline Impersonation & $\begin{array}{l}\text { A threat in which the attacker uses the personal data of } \\
\text { a credible user and is given the opportunity to access } \\
\text { and commit malicious acts. In the case of preventing } \\
\text { this attack, one has to take into account the correct } \\
\text { verification of user authentication. }\end{array}$ \\
\hline $\begin{array}{l}\text { Resource deprivation attacks } \\
\text { (Denial of Service - DoS) }\end{array}$ & $\begin{array}{l}\text { It is a type of malicious activity by which an attacker } \\
\text { exploits the vulnerability of certain applications or a } \\
\text { certain software part of user devices and sends a large } \\
\text { number of messages to user devices, in order to cause } \\
\text { congestion and make resources inaccessible to } \\
\text { legitimate users. }\end{array}$ \\
\hline Eavesdropping & $\begin{array}{l}\text { The user is passively listening to communication } \\
\text { between users and in that way may record the data } \\
\text { transmitted and come up with data. In order for this } \\
\text { attack to be prevented, one has to take into account the } \\
\text { confidentiality and integrity of data. }\end{array}$ \\
\hline Man-in-the-Middle Attack & $\begin{array}{l}\text { Man-In-The-Middle is an attack in which the attacker } \\
\text { secretly takes control of the communication channel } \\
\text { between the two communicating parties. } \\
\text { One way a MITM attack can compromise } \\
\text { communication is for an attacker to intercept a } \\
\text { message from the sender, modify it, and then forward } \\
\text { it to the recipient. }\end{array}$ \\
\hline Privacy sniffing & $\begin{array}{l}\text { In the device detection phase, an attacker can take } \\
\text { advantage of this D2D feature and find a legitimate } \\
\text { user. The use of a verified, anonymous identity is } \\
\text { required to prevent malicious activity. }\end{array}$ \\
\hline Jamming & $\begin{array}{l}\text { It is an attack that causes interference in the operation } \\
\text { of the D2D and disables the communication itself, } \\
\text { where the attacker performs interference via a signal } \\
\text { source that interferes with the communication signal. }\end{array}$ \\
\hline Malware Attacks & $\begin{array}{l}\text { Malicious software aimed at D2D pairs for the } \\
\text { collection of sensitive information. }\end{array}$ \\
\hline IP Spoofing & $\begin{array}{l}\text { Malicious nodes manipulate IP packages, especially } \\
\text { their headings. }\end{array}$ \\
\hline Free-Riding & $\begin{array}{l}\text { Malicious user equipment receives data from other } \\
\text { paired devices, but does not want to share the } \\
\text { downloaded resources with other devices, which } \\
\text { reduces the availability of the D2D system. }\end{array}$ \\
\hline Bandwidth Spoofing & $\begin{array}{l}\text { A malicious node gains unauthorised access to a } \\
\text { vulnerable legitimate user. }\end{array}$ \\
\hline Inference Attack & $\begin{array}{l}\text { Malicious activity of the attacker is realized through } \\
\text { logical or statistical study of transmission patterns } \\
\text { between devices, in order to obtain private information } \\
\text { of users (eg user behavior, location tracking). }\end{array}$ \\
\hline Location -spoofing & $\begin{array}{l}\text { A malicious device may emit a request message by } \\
\text { means of a wrong or defect information about location } \\
\text { to make it difficult to establish a D2D group. }\end{array}$ \\
\hline
\end{tabular}

The authors of the paper [25] have conducted research on the characteristics of DoS attacks on Android devices in the D2D network, and established that malicious devices can damage or even completely block the connecting of legitimate devices in the network.

Aside from the security threats listed above, it is important to mention that interleaving is an attack during which the attacker can change the authenticity of the response message sent to the base node, via a subnode. The attacker may add his/her own certificate and signature together with the message and start communication. This type of attack usually exists even in the case of a mutual authentication [26]. 


\subsection{Potential Security D2D Solutions}

In order to ensure security requirements and prevent security D2D threats presented above, it is necessary to improve the existing security mechanisms and think of new solutions that would be adjusted to the implementation of D2D technology in the 5th generation network. Security mechanisms have to efficient and flexible in order for them to be adjusted to various security threats. The abovementioned security challenges point to a need to establish a D2D communication model with a high level of security in the process of authentication, confidentiality, availability and integrity.

D2D cellular radio channels can be protected using cryptographic techniques consisting of symmetric key cryptography and public key cryptography. Symmetric key cryptography uses one key - a secret key that is shared between users, while public key cryptography involves the use of two different keys one key is used for encryption and that is the public key; the second key is used to decrypt and it is a secret key. Current cryptographic solutions require the involvement of a base station or a trusted third party whether it is one or the other key [20].

When it comes to the application of cryptographic techniques in order to protect the privacy of users of the D2D communication model, it is necessary to mention the cryptography of the elliptical curve, AES-128 and access points [31].

When compared to an earlier version of communication, where the key was being divided between two sides and distribution carried out via base station or another TTT, when D2D sides communicate directly without the network as the mediator, it is more difficult to share the key in a secure manner. Due to a great number of mobile devices, of various manufacturers and standards, it is difficult to integrate the hidden key into the devices prior to the communication [27].

At present, what is generally being treated is the aspect of managing keys in the context of $5 \mathrm{G}$, in D2D communication and the physical layer [21]. In fact, authors of the paper [28] suggest a key mechanism of distribution for D2D communication in $5 \mathrm{G}$, and a suppression of attacks targeted at the user "man in the middle"; the end scenario comprises two devices that belong to the same cellular network and coverage. The suggested protocols for the exchange of keys are based on the standard DiffieHellman algorithm based on the exchange and other easy cryptographic functions.
Apart from the Diffie-Hellman algorithm, the security of D2D communication is ensured through the usage of asymmetrical and by means of a combination of symmetrical and asymmetrical cryptographic algorithms such as River Shamir Adleman, Diffie Hellman (DH), Elliptic Curve Cryptography, and Elliptic Curve Cryptography in combination with Diffie Hellman (ECDH) and other combinations [26].

Dynamic Spectrum Access (DSA) technique is used with the aim of enhancing efficiency and may be implemented for various scenarios in D2D communication [29]. With co-operation, the energy consumption control and channel access are also important for the security of D2D communication. An optimal energy consumption and access to D2D link channel will contribute to an increase in the number of users of mobile services and the physical layer security [30].

\section{Conclusion}

Apart from massive MIMO systems, mm waves, green communication, software-defined network, virtualization of network functions and cloud computing, D2D communication is one of the key technologies that will enable applications and characteristics that are being presented to the $5 \mathrm{G}$ network, and to the greatest extent in terms of low latency, great transmission speed, coverage and reduced energy consumption.

D2D communication is a new wireless technology that by its activity reduces the load on the network and is a new tool for social networking, as it allows direct communication of devices without an indirect link with the network infrastructure. When it comes to the implementation of D2D communication in $5 \mathrm{G}$ network, we are talking about a local service, an implementation in cases of natural disasters and support of mutual connectivity of heterogenous objects. One of the scenarios is locating victims in cases of natural disasters or criminal activity by means of establishing a direct link with a police officer, etc.

Providing efficient security is the key question in D2D communication. This paper has offered a review of security-related issues when it comes to threats and offered solutions for improvement. However, in this research, we have come to a conclusion that there is a lack of global policy standards with regards to secure interaction of user equipment. Interoperability is difficult to achieve considering that the mechanisms used for authentication vary. 


\section{References}

[1]. Fodor, G., Dahlman, E., Mildh, G., Parkvall, S., Reider, N., Miklós, G., \& Turányi, Z. (2012). Design aspects of network assisted device-to-device communications. IEEE Communications Magazine, 50(3), 170-177.

[2]. Ioannou, I., Vassiliou, V., Christophorou, C., \& Pitsillides, A. (2020). Distributed artificial intelligence solution for D2D communication in $5 \mathrm{G}$ networks. IEEE Systems Journal, 14(3), 4232-4241.

[3]. Militano, L., Orsino, A., Araniti, G., Nitti, M., Atzori, L., \& Iera, A. (2016, September). Trusted D2D-based data uploading in in-band narrowband-IoT with social awareness. In 2016 IEEE 27th Annual International Symposium on Personal, Indoor, and Mobile Radio Communications (PIMRC) (pp. 1-6). IEEE.

[4]. Xu, W., Wu, D., Daneshmand, M., Liu, Y., \& Wang, M. (2015). A data privacy protective mechanism for WBAN. Wirel. Commun. Mob. Comput, 14, 421-430.

[5]. Haus, M., Waqas, M., Ding, A. Y., Li, Y., Tarkoma, S., \& Ott, J. (2017). Security and privacy in device-todevice (D2D) communication: A review. IEEE Communications Surveys \& Tutorials, 19(2), 10541079.

[6]. Tehrani, M. N., Uysal, M., \& Yanikomeroglu, H. (2014). Device-to-device communication in $5 \mathrm{G}$ cellular networks: challenges, solutions, and future directions. IEEE Communications Magazine, 52(5), 86-92.

[7]. Ansari, R. I., Chrysostomou, C., Hassan, S. A., Guizani, M., Mumtaz, S., Rodriguez, J., \& Rodrigues, J. J. (2017). 5G D2D networks: Techniques, challenges, and future prospects. IEEE Systems Journal, 12(4), 3970-3984.

[8]. Farzamiyan, A. H. (2019, March). A Survey on Device-to-Device Communication in $5 \mathrm{G}$ Wireless Networks. In 14th International Conference on Software Technologies (pp. 81-85).

[9]. Seok, B., Sicato, J. C. S., Erzhena, T., Xuan, C., Pan, Y., \& Park, J. H. (2020). Secure D2D communication for $5 \mathrm{G}$ IoT network based on lightweight cryptography. Applied Sciences, 10(1), 217.

[10]. Wang, C. X., Haider, F., Gao, X., You, X. H., Yang, Y., Yuan, D., ... \& Hepsaydir, E. (2014). Cellular architecture and key technologies for $5 \mathrm{G}$ wireless communication networks. IEEE communications magazine, 52(2), 122-130.

[11]. Asadi, A., Wang, Q., \& Mancuso, V. (2014). A survey on device-to-device communication in cellular networks. IEEE Communications Surveys \& Tutorials, 16(4), 1801-1819.

[12]. Mujkic, S., \& Kasapovic, S. (2018). Massive MIMO: design, application and challenges. B\&H Electrical Engineering, 12, 38-46.

[13]. Bello, O., \& Zeadally, S. (2014). Intelligent deviceto-device communication in the internet of things. IEEE Systems Journal, 10(3), 1172-1182.
[14]. Orsino, A., Araniti, G., Militano, L., Alonso-Zarate, J., Molinaro, A., \& Iera, A. (2016). Energy efficient IoT data collection in smart cities exploiting D2D communications. Sensors, 16(6), 836.

[15]. Umrao, S., Roy, A., \& Saxena, N. (2017). Device-todevice communication from control and frequency perspective: A composite review. IETE Technical Review, 34(3), 286-297.

[16]. Zhang, Y., Pan, E., Song, L., Saad, W., Dawy, Z., \& Han, Z. (2014). Social network aware device-todevice communication in wireless networks. IEEE Transactions on Wireless Communications, 14(1), 177-190.

[17]. Lin, X., Andrews, J. G., Ghosh, A., \& Ratasuk, R. (2014). An overview of 3GPP device-to-device proximity services. IEEE Communications Magazine, 52(4), 40-48.

[18]. Boccardi, F., Heath, R. W., Lozano, A., Marzetta, T. L., \& Popovski, P. (2014). Five disruptive technology directions for 5G. IEEE communications magazine, 52(2), 74-80.

[19]. Adnan, M. H., \& Ahmad Zukarnain, Z. (2020). Device-to-device communication in $5 \mathrm{G}$ environment: Issues, solutions, and challenges. Symmetry, 12(11), 1762 .

[20]. Jameel, F., Hamid, Z., Jabeen, F., Zeadally, S., \& Javed, M. A. (2018). A survey of device-to-device communications: Research issues and challenges. IEEE Communications Surveys \& Tutorials, 20(3), 2133-2168.

[21]. Noura, M., \& Nordin, R. (2016). A survey on interference management for device-to-device (D2D) communication and its challenges in 5G networks. Journal of Network and Computer Applications, 71, 130-150.

[22]. Gupta, A., \& Jha, R. K. (2015, May). Security threats of wireless networks: A survey. In International Conference on Computing, Communication \& Automation (pp. 389-395). IEEE.

[23]. Zhang, S., Wang, Y., \& Zhou, W. (2019). Towards secure 5G networks: A Survey. Computer Networks, 162, 106871.

[24]. Gandotra, P., Jha, R. K., \& Jain, S. (2017). A survey on device-to-device (D2D) communication: Architecture and security issues. Journal of Network and Computer Applications, 78, 9-29.

[25]. Hadiks, A., Chen, Y., Li, F., \& Liu, B. (2014, January). A study of stealthy denial-of-service attacks in Wi-Fi direct device-to-device networks. In 2014 IEEE 11th Consumer Communications and Networking Conference (CCNC) (pp. 507-508). IEEE.

[26]. Javed, Y., \& Khan, S. (2019). Major Security attacks in D2D Communication. Ubiquitous Comput. Commun. J., 1(1).

[27]. Wang, M., \& Yan, Z. (2017). A survey on security in D2D communications. Mobile Networks and Applications, 22(2), 195-208. 
[28]. Sedidi, R., \& Kumar, A. (2016, March). Key exchange protocols for secure device-to-device (D2D) communication in 5G. In 2016 Wireless Days (WD) (pp. 1-6). IEEE.

[29]. Fang, D., Qian, Y., \& Hu, R. Q. (2017). Security for $5 \mathrm{G}$ mobile wireless networks. IEEE Access, 6, 48504874.

[30]. Luo, Y., Cui, L., Yang, Y., \& Gao, B. (2015, October). Power control and channel access for physical-layer security of D2D underlay communication. In 2015 International Conference on Wireless Communications \& Signal Processing (WCSP) (pp. 1-5). IEEE.
[31]. Amin, R., Islam, S. H., Biswas, G. P., Khan, M. K., \& Kumar, N. (2015). An efficient and practical smart card based anonymity preserving user authentication scheme for TMIS using elliptic curve cryptography. Journal of medical systems, 39(11), 118.

[32]. Yang, Y., Zhang, Y., Dai, L., Li, J., Mumtaz, S., \& Rodriguez, J. (2016). Transmission capacity analysis of relay-assisted device-to-device overlay/underlay communication. IEEE transactions on industrial informatics, 13(1), 380-389.

[33]. Zhou, B., Hu, H., Huang, S. Q., \& Chen, H. H. (2013). Intracluster device-to-device relay algorithm with optimal resource utilization. IEEE transactions on vehicular technology, 62(5), 2315-2326. 